\title{
Nota \\ MÉTODOS DE FORMAÇÃO DE MUDAS DE MARACUJAZEIRO AMARELO
}

\author{
Marcelo Fontanetti Verdial'; Márcio Santos de Lima²; João Tessarioli Neto ${ }^{3 *}$; Carlos Tadeu dos \\ Santos Dias 4 ; Marcelo Trevizan Barbano ${ }^{5}$ \\ ${ }^{1}$ Pós-Graduando do Depto. de Produção Vegetal - USP/ESALQ. \\ ${ }^{2}$ Depto. de Produção Vegetal - UNESP/FCA, C.P. 237 - CEP: 18650-000 - Botucatu, SP. \\ ${ }^{3}$ Depto. de Produção Vegetal - USP/ESALQ, C.P. 09 - CEP: 13418-900 - Piracicaba, SP. \\ ${ }^{4}$ Depto. de Ciências Exatas - USP/ESALQ. \\ ${ }^{5}$ Centro de Solos e Recursos Agroambientais - IAC, C.P. 28 - CEP:13001-970 - Campinas, SP. \\ *Autor correspondente <jtessari@carpa.ciagri.usp.br>
}

RESUMO: Grande parte do sucesso de uma cultura está em implantá-la com mudas de alta qualidade. O objetivo do estudo foi avaliar métodos de formação de mudas de maracujazeiro amarelo quanto à qualidade das muda formada. Os tratamentos utilizados foram: 1- Mudas formadas em sacolas plásticas, preenchidas com terra adubada e misturada com esterco de curral curtido, com fertirrigação uma vez por semana; 2Mudas formadas em tubetes de plástico rígido, com substrato à base de casca de pinus e vermiculita, mantidos em bancada e recebendo fertirrigação uma vez por semana; 3- Idêntico ao tratamento 2, utilizando-se bandejas de poliestireno expandido; 4- Idêntico ao tratamento 3, sem fertirrigação, mas com uso de "floating" a cada dois dias; 5- Idêntico ao tratamento 4, com o uso de "floating" todo o período. O delineamento experimental foi completamente aleatorizado, com cinco tratamentos e quatro repetições. Na casa de vegetação, cada parcela foi composta por 64 plantas, sendo avaliadas semanalmente em oito plantas por parcela as seguintes variáveis: altura da planta; comprimento da raiz; matéria seca da parte aérea; matéria seca da parte radicular. O restante das mudas foi levado a campo para determinação da percentagem de pegamento e análise do desenvolvimento vegetativo inicial. Neste segundo estágio, cada parcela foi composta por cinco plantas. Sob casa de vegetação, o tratamento 5 apresentou os maiores valores para todas as variáveis, exceto para comprimento de raiz, onde houve superioridade do tratamento 1. Em campo, observou-se $100 \%$ de pegamento para todos os tratamentos, sendo que o tratamento 5 apresentou as maiores médias para altura de plantas.

Palavras-chave: 'floating', maracujá, muda, condicionamento

\section{METHODS OF YELLOW PASSION FRUIT SEEDLING PRODUCTION}

ABSTRACT: The main part of a successful production of passion fruit is dependent on seedling quality. The present research had the objective of comparing methods of passion fruit seedling production. The treatments were: 1- seedlings formed in plastic bags, filled with fertilized soil, and fertirrigated weekly; 2- seedling formed in rigid plastic tubes, filled with commercial substract, kept on a bench and receiving the same fertirrigation as treatment 1; 3- As treatment 2, but the containers were polystyrene trays; 4- As treatment 3, but the seedlings were not fertirrigated, and were placed into a "floating" system every two days; 5 - Same as treatment 4, but the trays were kept throughout the period inside the "floating" system. The experimental design was completely randomized with five treatments and four replications. In the greenhouse, the experimental arrangement was composed of 64 plants. Each plot of eight plants was evaluated weekly, for the following variables: 1- plant height; 2- root length; 3- shoot dry weight; 4- root dry weight. After this period, the remaining seedlings were taken to the field for plant survival (\%) and development analysis. In this second stage, the experimental arrangement was composed of 5 plants per plot. In the greenhouse, the trays kept in the floating system (treatment 5) showed the highest averages for all the variables, except for root length, for which the highest average was found for seedlings kept in plastic bags (treatment 1). In the field, a 100\% rate of succeeds was observed for all treatments, and plants from treatment number 5 showed the highest average values for plant height.

Key words: 'floating', passion fruit, seedling, container

\section{INTRODUÇÃO}

A cultura do maracujazeiro no Brasil se expandiu em ritmo acelerado desde o início da década de 1970. Até então, o Brasil não figurava entre os maiores produtores do mundo, porém, a partir daí a cultura ganhou grande impulso, principalmente pela crescente exportação de suco concentrado (São José, 1994).
No Brasil e em muitos outros países, a multiplicação do maracujazeiro amarelo é feita principalmente através de sementes, embora também possa ser realizada através de enxertia e estaquia (São José, 1994).

A produção de mudas de alta qualidade torna-se estratégica para quem deseja tornar mais competitiva sua produção, e aumentar a exportação. Considera-se que $60 \%$ do sucesso de uma cultura está em implantá-la com mudas 
de alta qualidade (Minami et al.,1994). Uma boa muda de maracujá deve ter pelo menos $25 \mathrm{~cm}$ de altura, deve ser sadia, ter 4 a 5 folhas verdadeiras vigorosas e estar emitindo a primeira gavinha (São José, 1994).

Dentre os vários recipientes existentes no mercado, o mais utilizado para a produção de mudas de maracujá é a sacola plástica de $10 \times 25 \mathrm{~cm}$ ou $18 \times 30 \mathrm{~cm}$, comumente usadas na produção de mudas de café de um ano.

Pode-se ainda dispor-se de tubetes ou de bandejas de poliestireno expandido (isopor) para a formação das mudas, o que tem sido utilizado principalmente por grandes empresas produtoras de mudas. De acordo com Tessarioli Neto (1995), a semeadura em recipientes é atualmente a forma mais empregada na produção de mudas de maracujazeiro. As vantagens do sistema de produção de mudas em recipientes são múltiplas e justificam plenamente sua adoção. Dentre outras, tem-se: maior precocidade; menor possibilidade de contaminação fitopatogênica; melhor controle ambiental; melhor aproveitamento das sementes e da área de produção de mudas; menor "stress" no transplante. Estas vantagens se manifestam durante a produção das mudas e, posteriormente, na fase de transplante para o campo de produção comercial.

Tessarioli Neto (1995) ainda cita que as bandejas de isopor apresentam vantagem sobre os tubetes, por serem excelentes isolantes térmicos, e permitirem melhor desenvolvimento das mudas, mesmo em condições extremas de temperatura.

São José (1994) refere-se a problemas com a produção de mudas em tubetes, relacionados, principalmente, com o substrato, cujos nutrientes são reduzidos ou totalmente esgotados em poucas semanas, por ação de lavagem. Assim, recomenda-se regar as mudas com uma solução de adubo solúvel, iniciando-se após $025^{\circ}$ dia (quando as plantas apresentarem cerca de $5 \mathrm{~cm}$ de altura). Além da pulverização com a solução de adubo líquido, as mudas devem receber um tratamento preventivo contra doenças fúngicas, e, quando necessário, poderão ser usados inseticidas.

Deve-se atentar para a nutrição suplementar das mudas quando estas são produzidas em tubetes ou bandejas de isopor, devido a escassez de substrato dos recipientes. Desse modo, os autores sugerem que a irrigação deva ser feita com solução nutritiva e em alta freqüência (Souza \& Meletii, 1997). Segundo São José (1994), pode-se forçar o crescimento das mudas com uma adubação nitrogenada, via irrigação, feita semanalmente com uma solução $0,5 \%$ a $1,0 \%$ de uréia.

Outra técnica que pode ser usada com o intuito de forçar o crescimento das mudas, é o condicionamento nutricional com a utilização do "floating". O "floating" consiste em um tanque de aproximadamente $30 \mathrm{~cm}$ de altura, tendo largura e comprimento suficientes para acomodar o número de bandejas desejadas, preenchidas com uma solução de água e adubo solúvel, onde as bandejas de poliestireno expandido são colocadas e ficam boiando.

De acordo com Verdial et al. (1998), o condicionamento nutricional com a utilização do "floating", em mudas de pimentão, proporcionou maior desenvolvimento, além de permitir o controle do desenvolvimento das mesmas, mostrando-se uma técnica viável e de fácil utilização.

Verdial et al. (1999), estudando a influência do uso do "floating" na produção de mudas de tomate, concluíram que as bandejas que ficaram dentro do "floating" em todo 0 período experimental produziram mudas de maior alturas e massa de matéria fresca das partes aérea e da raiz superiores.

O presente trabalho teve como objetivo comparar métodos de formação de mudas de maracujazeiro amarelo, incluindo a técnica do "floating".

\section{MATERIAL E MÉTODOS}

O experimento foi realizado dentro de uma estufa do tipo túnel alto, pertencente à Escola Superior de Agricultura "Luiz de Queiroz", USP, Piracicaba, no período de 05 de março a 05 de junho de 1997.

Foram utilizadas sementes de maracujá amarelo "Golden Star", apresentando pureza de 99\% e garantia de germinação mínima de $75 \%$.

Os tratamentos foram os seguintes: 1- Mudas formadas em sacolas plásticas de $10 \times 25 \mathrm{~cm}$, preenchidas com uma mistura de terra e esterco de curral curtido, previamente adubada com $4 \mathrm{~kg}$ de superfosfato simples e $0,75 \mathrm{~kg}$ de cloreto de potássio por metro cúbico. Neste tratamento, as mudas ainda receberam fertirrigação semanalmente; 2- Mudas formadas em tubetes de plástico rígido de $15 \mathrm{~cm}$ de comprimento, mantidos sob bancadas a $0,8 \mathrm{~m}$ do solo, preenchidos com um substrato à base de casca de pinus e vermiculita. Este tratamento também recebeu fertirrigação semanalmente; 3- O mesmo tratamento 2, sendo que neste caso os recipientes foram bandejas de isopor de 128 células; 4- Mudas formadas em bandejas de isopor de 128 células, preenchidas com 0 mesmo substrato do tratamento 2, colocadas alternadamente 2 dias em "floating" e 2 dias sobre bancada a 0,8m do solo; 5- Mudas formadas em bandejas de isopor de 128 células, preenchidas com o mesmo substrato do tratamento 2, colocadas desde a semeadura dentro do "floating".

Utilizou-se a mesma solução de adubo para fertirrigação e para o enchimento do "floating". A concentração de adubo nesta solução foi de $1,0 \mathrm{~g} \mathrm{~L}^{-1}$ sendo que este adubo apresentava os seguintes nutrientes nas respectivas proporções: Nitrogênio (20\%), $\mathrm{P}_{2} \mathrm{O}_{5}(10 \%), \mathrm{K}_{2} \mathrm{O}(20 \%), \mathrm{B}$ $(0,02 \%)$, Cu $(0,05 \%)$, Fe $(0,10 \%), \mathrm{Mn}(0,05 \%)$, Mo $(0,0005 \%)$ e Zn $(0,05 \%)$.

Após $028^{\circ}$ dia do início da germinação, as seguintes variáveis foram avaliadas, a cada sete dias durante o período de um mês: altura da planta (ALT1), que foi medida do colo até o ápice da planta, expressa em centímetros; comprimento da raiz (COMP), expresso em milímetros; matéria seca da parte aérea (MSPA), expresso em gramas; massa seca da parte radicular (MSPR), também expresso em gramas. 
Após este período, as mudas foram levadas para o campo a fim de se avaliar a percentagem de pegamento e o desenvolvimento inicial, determinados pela altura das plantas no campo, expressa em centímetros (ALT2), e medida a cada sete dias durante o período de um mês.

Na primeira etapa do experimento, o delineamento experimental foi completamente aleatorizado, com cinco tratamentos e quatro repetições, havendo 64 plantas por parcela. Semanalmente, foram analisadas oito plantas de cada parcela. O restante das mudas foi levada a campo para determinação da percentagem de pegamento $e$ análise do desenvolvimento inicial das mesmas. $\mathrm{Na}$ segunda etapa, o delineamento experimental também foi completamente aleatorizado, havendo cinco tratamentos e quatro repetições, com cinco plantas por parcela.

A análise de variância foi obtida pelo teste $F$ de Snedecor, e as diferenças das médias dos tratamentos foram comparadas pelo teste t-Student a $1 \%$. Os dados foram transformados utilizando-se do método da potência ótima de BOX-COX (Atikinson, 1985).

\section{RESULTADOS E DISCUSSÃO}

Para a variável MSPR pode-se notar, desde a primeira avaliação (28을 dia), a superioridade do tratamento 5 em relação aos demais tratamentos (TABELA 1). Na a última avaliação (49ํำ dia), o tratamento 1 equiparou-se ao tratamento 5, superiores aos demais, a 0,01 de probabilidade.

Para a variável MSPA, o tratamento 5 também apresentou as maiores médias a partir da avaliação do $35^{\circ}$ dia após o início da germinação. $\mathrm{Na}$ avaliação do $42^{\circ}$ dia, o tratamento 4 não apresentou diferença significativa em relação ao tratamento 5 para esta característica. As mudas formadas em tubetes e badejas de isopor sem o uso do "floating" (tratamentos 2 e 3 respectivamente) apresentaram as menores médias para MSPA, ao final do período de avaliação.

Para a variável ALT1, tratamento 5 apresentou as maiores médias ao longo de todo período, diferenciandose estatisticamente das demais. Entretanto, o crescimento das mudas do tratamento 5 foi exagerado e demasiadamente rápido, provocando o estiolamento das plantas. Assim, apesar do tratamento 4 não ter sido aquele que apresentou as maiores médias, foi o que resultou nas melhores mudas, pois favoreceu o crescimento das plântulas, sem, contudo, provocar o estiolamento das mesmas. Foi observado ainda, que os tratamentos 2 e 3 foram novamente inferiores em altura de planta.

Para as variáveis MSPR, MSPA E ALT1, foi demonstrando incremento no desenvolvimento das mudas em função do uso do "floating", devido à pronta disponibilidade de nutrientes solúveis às plantas. Estes dados conferem com aqueles obtidos por Verdial et al. (1998 e 1999), para as culturas de pimentão e tomate, respectivamente.
TABELA 1 - Valores médio de peso da matéria seca da raiz (MSPR), peso da matéria seca da parte aérea (MSPA), altura de plantas (ALT1) e comprimento de raízes (COMP) de mudas de maracujazeiro amarelo produzidas sob diferentes sistemas, em casa de vegetação, Piracicaba, 1997.

\begin{tabular}{|c|c|c|c|c|}
\hline & \multicolumn{4}{|c|}{ Tempo em semanas } \\
\hline & 1 & 2 & 3 & 4 \\
\hline & \multicolumn{4}{|c|}{$\mathrm{MSPR} \mathrm{MSPR}^{0,1}(\mathrm{~g})$} \\
\hline Sacolas plásticas & $0,794 \mathrm{c}$ & $0,837 \mathrm{~b}$ & $0,851 \mathrm{~b}$ & $0,970 \mathrm{a}$ \\
\hline $\begin{array}{l}\text { Tubetes com } \\
\text { fertirrigação }\end{array}$ & $0,808 \mathrm{c}$ & $0,822 \mathrm{~b}$ & $0,875 \mathrm{~b}$ & $0,912 b$ \\
\hline $\begin{array}{l}\text { Bandejas com } \\
\text { fertirrigação }\end{array}$ & $0,837 \mathrm{~b}$ & $0,837 \mathrm{~b}$ & $0,884 \mathrm{~b}$ & $0,917 b$ \\
\hline $\begin{array}{l}\text { Floating } 2 \text { dias } \\
\text { sim, } 2 \text { dias não }\end{array}$ & $0,860 \mathrm{~b}$ & $0,822 \mathrm{~b}$ & $0,868 \mathrm{~b}$ & $0,927 \mathrm{~b}$ \\
\hline $\begin{array}{l}\text { Floating } \\
\text { constantemente }\end{array}$ & 0,932 a & 0,932 a & $1,021 \mathrm{a}$ & $1,005 \mathrm{a}$ \\
\hline $\mathrm{F}$ & 30,37 & & & \\
\hline \multirow[t]{2}{*}{ C.V.\% } & $2,64 \%$ & & & \\
\hline & \multicolumn{4}{|c|}{$\mathrm{MSPA} \mathrm{MSPA}^{0,2}(\mathrm{~g})$} \\
\hline Sacolas plásticas & 0,803 a & $0,880 \mathrm{~b}$ & $0,948 \mathrm{~b}$ & $1,153 \mathrm{~b}$ \\
\hline $\begin{array}{l}\text { Tubetes com } \\
\text { fertirrigação }\end{array}$ & $0,809 a$ & $0,830 \mathrm{~b}$ & $0,888 \mathrm{~b}$ & $0,886 \mathrm{~d}$ \\
\hline $\begin{array}{l}\text { Bandejas com } \\
\text { fertirrigação }\end{array}$ & $0,797 a$ & $0,809 \mathrm{~b}$ & $0,928 \mathrm{~b}$ & $0,988 \mathrm{c}$ \\
\hline $\begin{array}{l}\text { Floating } 2 \text { dias } \\
\text { sim, } 2 \text { dias não }\end{array}$ & $0,830 \mathrm{a}$ & 0,878 a & 1,072 a & $1,197 \mathrm{~b}$ \\
\hline $\begin{array}{l}\text { Floating } \\
\text { constantemente }\end{array}$ & $1,057 \mathrm{a}$ & 1,162 a & $1,162 \mathrm{a}$ & $1,424 \mathrm{a}$ \\
\hline $\mathrm{F}$ & 83,28 & & & \\
\hline \multirow[t]{2}{*}{ C.V.\% } & $4,25 \%$ & & & \\
\hline & \multicolumn{4}{|c|}{$\mathrm{ALT1}=\mathrm{ALT1}^{0,4}(\mathrm{~cm})$} \\
\hline Sacolas plásticas & $1,860 \mathrm{~b}$ & $1,930 \mathrm{~b}$ & $2,059 \mathrm{c}$ & $2,450 \mathrm{c}$ \\
\hline $\begin{array}{l}\text { Tubetes com } \\
\text { fertirrigação }\end{array}$ & $1,563 \mathrm{~b}$ & $1,619 \mathrm{c}$ & $1,835 \mathrm{c}$ & $1,856 \mathrm{e}$ \\
\hline $\begin{array}{l}\text { Bandejas com } \\
\text { fertirrigação }\end{array}$ & $1,648 b$ & $1,691 \mathrm{c}$ & $1,921 \mathrm{c}$ & $2,073 \mathrm{~d}$ \\
\hline $\begin{array}{l}\text { Floating } 2 \text { dias } \\
\text { sim, } 2 \text { dias não }\end{array}$ & $1,730 \mathrm{~b}$ & $1,931 \mathrm{~b}$ & $2,446 \mathrm{~b}$ & $2,715 b$ \\
\hline $\begin{array}{l}\text { Floating } \\
\text { constantemente }\end{array}$ & $2,339 a$ & $2,761 \mathrm{a}$ & $3,277 \mathrm{a}$ & $3,441 \mathrm{a}$ \\
\hline $\mathrm{F}$ & 176,58 & & & \\
\hline \multirow[t]{2}{*}{ C.V.\% } & $3,76 \%$ & & & \\
\hline & \multicolumn{4}{|c|}{$\mathrm{COMP}=\mathrm{COMP}^{-0,5}(\mathrm{~cm})$} \\
\hline Sacolas plásticas & $0,420 \mathrm{~b}$ & $0,292 \mathrm{c}$ & $0,285 \mathrm{c}$ & $0,256 \mathrm{~d}$ \\
\hline $\begin{array}{l}\text { Tubetes com } \\
\text { fertirrigação }\end{array}$ & $0,304 \mathrm{c}$ & $0,292 \mathrm{c}$ & $0,294 \mathrm{c}$ & $0,288 \mathrm{c}$ \\
\hline $\begin{array}{l}\text { Bandejas com } \\
\text { fertirrigação }\end{array}$ & $0,422 \mathrm{~b}$ & 0,408 a & $0,423 a$ & $0,408 a$ \\
\hline $\begin{array}{l}\text { Floating } 2 \text { dias } \\
\text { sim, } 2 \text { dias não }\end{array}$ & $0,444 a$ & $0,415 \mathrm{a}$ & 0,408 a & $0,408 \mathrm{a}$ \\
\hline $\begin{array}{l}\text { Floating } \\
\text { constantemente }\end{array}$ & $0,272 \mathrm{~d}$ & $0,308 \mathrm{~b}$ & $0,365 \mathrm{~b}$ & $0,348 \mathrm{~b}$ \\
\hline$F$ & 182,37 & & & \\
\hline C.V.\% & $2,64 \%$ & & & \\
\hline
\end{tabular}

Médias seguidas pela mesma letra (coluna) não diferem pelo teste t-Student, a $1 \%$.

Dados transformados pelo método da potência ótima de BOX COX. 
Quando foram comparados os tratamentos 2 e 3 com o tratamento 1, notou-se a superioridade do tratamento 1 (mudas formadas sacolas plásticas) em relação aos outros dois (mudas formadas em tubetes e bandejas de isopor, respectivamente), sendo que dentre os três, as menores médias foram observadas no tratamento 2. Este fato já era esperado de acordo com citações de São José (1994) e Souza \& Meletti (1997). O menor volume de substrato nos tubetes e nas bandejas de isopor em relação às sacolas plásticas explica o menor desenvolvimento das mudas nesses recipientes, já que, comparativamente, a quantidade nutrientes é menor e tende a lixiviar mais rapidamente pela ação da irrigação.

Para a variável COMP, o uso do "floating" no tratamento 5 proporcionou melhor crescimento inicial da parte radicular das mudas, representado na avaliação do $28^{\circ}$ dia, onde este tratamento diferenciou-se estatisticamente dos demais, com as maiores médias. Entretanto, a limitação de espaço nas células das bandejas impediu que esse tratamento continuasse a ser superior nas avaliações que se sucederam.

Assim, na última avaliação de comprimento de raízes (49º dia), o tratamento 1 apresentou as maiores médias, superando e diferenciando-se dos demais tratamentos, uma vez os saquinhos plásticos ofereceram um maior volume de substrato para o crescimento radicular.

Já os tratamentos 3 e 4 apresentaram as menores médias e não tiveram diferenças significastes. Este fato explica-se pelo menor tamanho dos recipientes nesses tratamentos, que impediram um maior desenvolvimento radicular. O tratamento 5 , cuja bandeja de isopor ficou durante todo o período dentro do "floating", apresentou médias maiores que dos tratamentos 3 e 4 pelo fato de possibilitar que as raízes se desenvolvessem além do espaço das células.

Não foram observadas diferenças significativas para percentagem de pegamento de mudas no campo, sendo que todos os tratamento apresentam $100 \%$ de pegamento.

Quanto à altura de plantas em campo (TABELA 2) o tratamento 5 foi aquele que apresentou as maiores médias ao longo de todo período observado, diferenciandose estatisticamente dos demais. Já o tratamento 3 foi aquele que apresentou as menores médias. Em todas as avaliações permaneceram as mesmas diferenças estatísticas entre as médias dos tratamentos. Isto evidencia que, para o maracujá amarelo, o tamanho da muda influencia o desenvolvimento inicial das mesmas, sendo que mudas maiores tendem a formar plantas mais desenvolvidas.

\section{CONCLUSÕES}

- Mudas formadas dentro do "floating" desde a semeadura obtiveram melhor desempenho para a maioria das variáveis estudadas, exceto para comprimento de raiz, onde destacou-se o uso de sacolas plásticas. Entretanto, o uso contínuo do "floating" ocasionou o estiolamento das mudas.
TABELA 2 - Valores médios de altura de plantas em campo (ALT2) de mudas de maracujá amarelo produzidas sob diferentes sistemas, Piracicaba, 1997.

\begin{tabular}{lcccc}
\hline & \multicolumn{4}{c}{ Tempo em semanas } \\
\cline { 2 - 5 } & 1 & 2 & 3 & 4 \\
\hline $\begin{array}{l}\text { Sacolas } \\
\text { plásticas }\end{array}$ & 2,384 b & 2,467 b & 2,648 b & 2,939 b \\
$\begin{array}{l}\text { Tubetes com } \\
\text { fertirrigação }\end{array}$ & 1,903 c & 1,997 c & 2,106 c & 2,311 c \\
$\begin{array}{l}\text { Bandejas com } \\
\text { fertirrigação }\end{array}$ & 1,710 d & 1,800 d & 1,863 d & $2,073 d$ \\
$\begin{array}{l}\text { Floating 2 dias } \\
\text { sim, 2 dias não }\end{array}$ & 2,427 b & 2,568 b & 2,660 b & 3,033 b \\
$\begin{array}{l}\text { Floating } \\
\text { constantemente }\end{array}$ & 2,996 a & 3,070 a & 3,211 a & 3,734 a \\
\hline \begin{tabular}{l} 
F \\
\hline C.V.\%
\end{tabular} & \multicolumn{4}{c}{111,36} \\
\hline
\end{tabular}

Médias seguidas pela mesma letra (coluna) não diferem pelo teste t-Student, a $1 \%$.

Dados transformados pelo método da potência ótima de BOX COX.

- As mudas formadas em tubetes e bandejas de isopor, com apenas uma fertirrigação por semana, mostraram-se inferiores quando comparadas àquelas formadas em saquinhos plásticos com a mesma quantidade de fertirrigação.

- A utilização do sistema de "floating" no condicionamento do desenvolvimento de mudas de maracujazeiro amarelo mostrou-se experimentalmente viável, permitindo o controle do desenvolvimento das mesmas e proporcionando um maior desenvolvimento inicial em campo.

\section{REFERÊNCIAS BIBLIOGRÁFICAS}

ATINKINSON, A.C. Plots, transformations, and regression. Clarendon Press: Oxford, 1985. 282p. (Oxford Statistical Science Series).

MINAMI, K.; TESSARIOLI NETO, J.; PENTEADO, S.R.; ESCARPARI FILHO, J.A. Produção de mudas hortícolas de alta qualidade. Piracicaba: ESALQ/SEBRAE, 1994. 155p. SOUZA, J. S. I. de; MELETTI, L. M. M. Maracujá: espécies, variedades e cultivo. Piracicaba: FEALQ, 1997. 180p.

SÃO JOSÉ,A.R. A cultura do maracujazeiro: produção e mercado. Vitória da Conquista: DFZ/UESB,1994. 255p.

TESSARIOLI NETO, J. Recipientes, embalagens e acondicionamentos de mudas de hortaliças. In: MINAMI,K. Produção de mudas de alta qualidade em horticultura. São Paulo: T.A.QUEIROZ, 1995. cap. 4, p.59-64.

VERDIAL, M.F; IWATA, A.Y; LIMA, M.S.de ; TESSARIOLI NETO, J.; Influência do "floating" no condicionamento e crescimento de mudas de pimentão (Capsicum annum L.). Scientia Agricola, v.55, p.25-28, 1998.

VERDIAL, M.F; IWATA, A.Y; LIMA, M.S.de ; TESSARIOLI NETO, J; TAVARES, M;. Influência do sistema de "floating" no condicionamento do crescimento mudas de tomateiro (Lycopersicon esculentum Mill.). Revista de Agricultura, v.74, p.107-115, 1999.

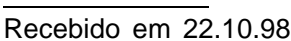

